\title{
Randomized clinical trial of multimodal physiotherapy treatment compared to overnight lidocaine ointment in women with provoked vestibulodynia: Design and methods
}

Mélanie Morina, , , Chantale Dumoulinb, Sophie Bergeronc, Marie-Hélène Mayrandd, Samir Khalifée, Guy Waddellf, Marie-France Duboisg, Provoked vestibulodynia (PVD) Study Group1,

\begin{abstract}
Provoked vestibulodynia (PVD) is a highly prevalent and debilitating condition yet its management relies mainly on non-empirically validated interventions. Among the many causes of PVD, there is growing evidence that pelvic floor muscle (PFM) dysfunctions play an important role in its pathophysiology. Multimodal physiotherapy, which addresses these dysfunctions, is judged by experts to be highly effective and is recommended as a first-line treatment. However, the effectiveness of this promising intervention has been evaluated through only two small uncontrolled trials. The proposed bi-center, single-blind, parallel group, randomized controlled trial (RCT) aims to evaluate the efficacy of multimodal physiotherapy and compare it to a frequently used first-line treatment, topical overnight application of lidocaine, in women with PVD. A total of 212 women diagnosed with PVD according to a standardized protocol were eligible for the study and were randomly assigned to either multimodal physiotherapy or lidocaine treatment for 10 weeks. The primary outcome measure is pain during intercourse (assessed with a numerical rating scale). Secondary measures include sexual function, pain quality, psychological factors (including pain catastrophizing, anxiety, depression and fear of pain), PFM morphology and function, and patients' global impression of change. Assessments are made at baseline, post-treatment and at the 6-month follow-up. This manuscript presents and discusses the rationale, design and methodology of the first RCT investigating physiotherapy in comparison to a commonly prescribed first-line treatment, overnight topical lidocaine, for women with PVD.
\end{abstract}

Keywords

Dyspareunia; Lidocaine; Pelvic floor muscles; Physical therapy; Provoked vulvodynia

\section{Introduction}

Vulvodynia, a highly neglected chronic pain condition, has a 7-8\% prevalence [1]. Considered as the leading cause of pre-menopausal vulvodynia, provoked vestibulodynia (PVD) is characterized by an acute pain at the entry of the vagina during application of pressure or attempted vaginal penetration [2]. PVD is reported to disrupt personal lives, severely affect sexual function and negatively impact quality of life [3] and [4]. It is also related to relationship problems and psychological distress [5]. Poorly understood, often misdiagnosed or ignored, such pain leads to a high personal cost for patients and substantial financial cost for society. Women multiply their medical visits hoping to find relief and rely mainly on non-evidence-based, ineffective interventions [6]. 
The exact etiology of PVD remains unclear. Proposed biomedical factors include vaginal infections [7], genetic or immune factors [8], hormonal factors [9], or the proliferation of nociceptors and sensitization [10]. Pelvic floor muscle (PFM) dysfunctions may also play an important role [11], [12] and [13]. It has been reported that women with PVD have heightened PFM tone as well as inferior PFM strength, coordination and endurance compared to asymptomatic women [11], [12] and [13]. Targeting these dysfunctions, multimodal physiotherapy treatment includes education, biofeedback, manual and insertion techniques [14]. This intervention is judged highly effective by vulvodynia specialists [15] and is listed as a first-line treatment for vulvodynia in clinical guidelines [16] and [17]. Individual physiotherapy modalities have been shown to reduce pain significantly in women with PVD [18], [19] and [20]. A randomized controlled trial (RCT) showed a $35 \%$ pain reduction during intercourse following PFM biofeedback [18]. Manual therapy and auto-insertion techniques also appear to reduce pain [19] and [20]. However, it should be emphasized that these isolated modalities do not realistically represent physiotherapy in a clinical setting as described by Hartmann et al. [14] and may yield only partial efficacy compared to a multimodal physiotherapy. To date, the efficacy of multimodal physiotherapy has not been evaluated in a RCT. Only three case reports in women with dyspareunia [21], [22] and [23] and two small uncontrolled studies [24] and [25] have investigated the effectiveness of combined treatment. One retrospective study involving 35 women with PVD and one prospective uncontrolled trial in 13 women showed an overall reduction of pain in $71-77 \%$ of women, which exceeds the reported effectiveness of the modalities taken separately. Improvements in sexual function and psychological variables are also reported [24] and [25]. It is therefore important and timely to evaluate the efficacy of the promising multimodal physiotherapy treatment, reflecting current clinical practice, in a RCT design.

Recommended as a first-line treatment in clinical guidelines, topical lidocaine was found to be one of the most commonly prescribed treatments for vulvodynia [15], [16] and [17]. The hypothesized mechanism of action is that repeated application of this anesthetic could act on nociceptor proliferation and sensitization [10]. Reducing their hyperexcitability is presumed to yield long-lasting pain reduction [10] and [26]. In a prospective uncontrolled study, Zolnoun et al. [26] showed that overnight use of $5 \%$ lidocaine ointment applied for eight weeks significantly reduced pain and improved sexual function in 59\% of participants with PVD. Other studies using lidocaine showed improvement in pain and sexual function. However, when comparing lidocaine to other treatments, its efficacy was found nonsignificantly different than biofeedback in the study of Danielsson et al. [27] and desipramine and/or placebo in the study of Foster et al. [28]. The latter's posology and technique of application differed from those of Zolnoun et al. [26], which may explain the lower efficacy. In fact, Danielsson et al. [27] instructed women to apply lidocaine $2 \%$ and $5 \%$ five to seven times per day while Foster et al. [28] recommended four-five daily applications of lidocaine $2 \%$ or $5 \%$ diluted in hydrating cream. Overnight lidocaine $5 \%$ may represent a better treatment option but its efficacy has never been investigated using a rigorous RCT design. 
We designed a bi-center RCT to gather convincing evidence about a recommended intervention (multimodal physiotherapy) and compare it to another frequently used first-line intervention (overnight lidocaine). This paper discusses the rationale, design and methodology as well as challenges encountered during study implementation.

\section{Methods}

\subsection{Design overview}

This study consists of a single-blind, parallel-group RCT conducted at two Canadian university hospital centers. The overall design is shown in Fig. 1 . The primary aim is to compare the efficacy of multimodal PFM physiotherapy to overnight topical lidocaine for reducing pain during sexual intercourse in women with PVD. Considering the reported effectiveness of the two treatments [24], [25] and [26] and the opinion of experts, the main hypothesis is that, in comparison to lidocaine, women having physiotherapy will show a greater reduction of pain (post-treatment and at 6-month follow-up compared to baseline assessment). Secondary aims include comparing the effects of the two treatments on: 1) pain quality (affective, sensory and evaluative components); 2) sexual function; 3) psychological variables (catastrophizing, anxiety, depression, fear of pain); 4) PFM morphology and function and 5) patients' global impression of change.

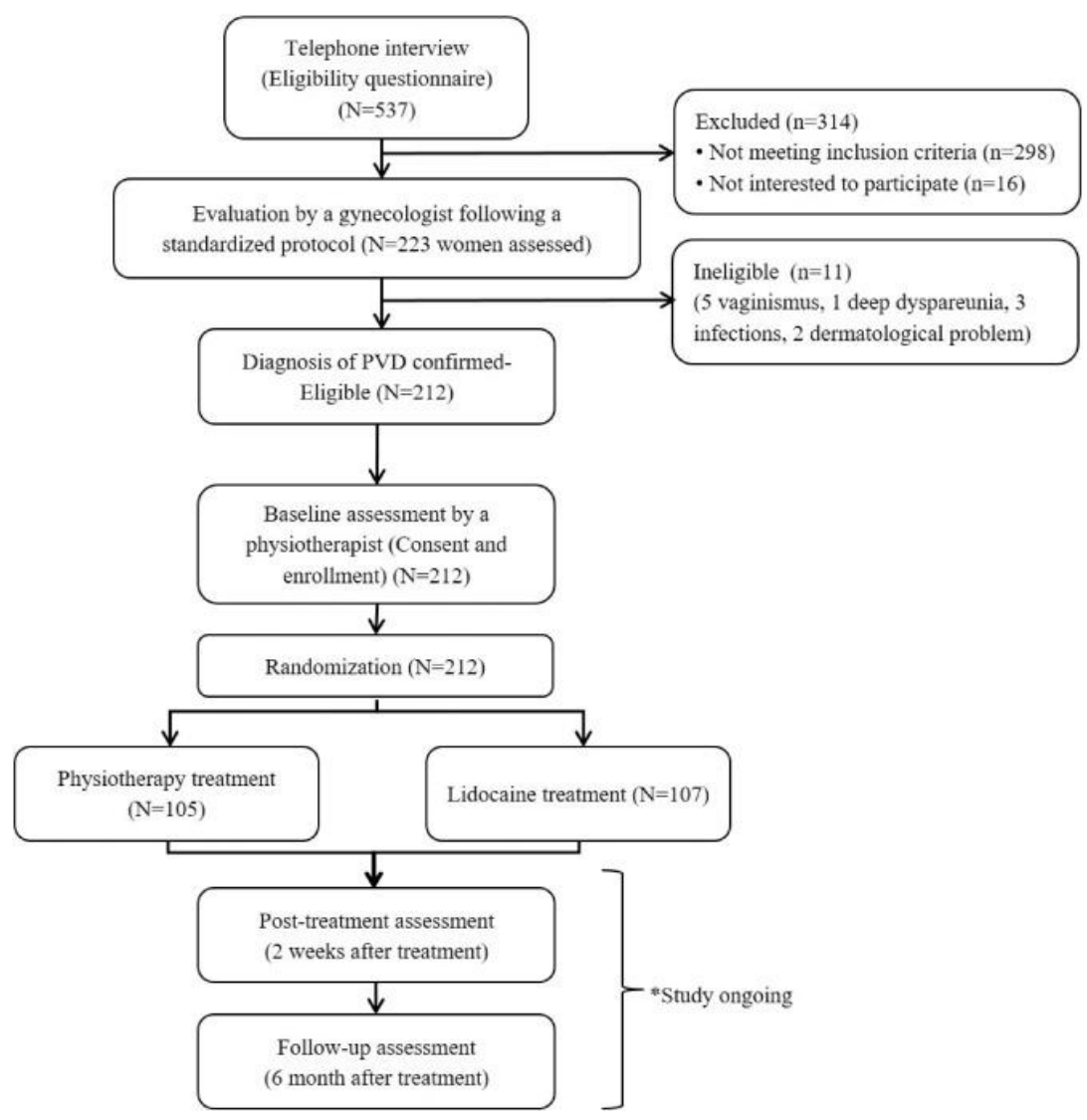

Fig. 1. Study design flow diagram. 


\subsection{Participants}

A total of 212 nulliparous women with PVD, 18 to 45 years old, were recruited for this study. To be included, women had to report pain in the vestibule area at an average intensity of 5 or more on the Numerical Rating Scale (NRS) during penetration, which is indicative of moderate to severe pain [29]. Both primary (i.e. pain appeared th the first sexual intercourse) and secondary (i.e. pain occurred after successful unpainful intercourse) subtypes were included in the study. In order to confirm the PVD diagnosis, all women underwent a medical history interview and a physical examination including a standardized pelvic examination performed by a gynecologist of our team [18]. This evaluation followed the diagnostic criteria defined by Friedrich [2] and more recently modified by Bergeron et al. [30]: 1) pain in the vestibule following touch or an attempted vaginal penetration; 2) acute pain during the cotton-swab test which consists in applying pressure following a random order to the vulvar vestibule. The inter-rater reliability of this diagnostic method has been demonstrated [30]. Vulvar pain occurring in the absence of an underlying recognizable disease and provoked spontaneously as a result of physical contact can be classified as PVD [31]. Therefore, our assessment procedure aimed to rule out any other specific neuropathology, atrophic vaginitis, dermatoses such as lichen sclerosus, or pathogens such as culture- or smear-proven Candida species, Gardnerella, Trichomonas, herpes simplex, gonorrhea and chlamydia. More details about the inclusion and exclusion criteria are presented in Table 1.

Table 1.

Inclusion and exclusion criteria.

\section{Inclusion criteria}

Aged 18 to 45 years;

experience moderate to severe pain in at least $90 \%$ of attempted sexual intercourse with an average intensity of $5 / 10$ on a numeric rating scale of pain intensity;

pain limited to the vestibule during vaginal intercourse and other activities exerting pressure (tampon insertion, tight jeans or pants, cycling, horseback riding);

$-$

presence of PVD for at least 6 months and diagnosed according to the standardized gynecological examination protocol by one of the team gynecologists; and

have a stable sexual partner (sexual activity should include some attempted vaginal penetrations in order to evaluate pain intensity).

\section{Exclusion criteria}


dyspareunia) and constant, spontaneous vulvar pain;

$-$

vaginismus (as defined by Diagnostic and Statistical Manual of Mental Disorders IV [32]);

$-$

current or previous pregnancy that lasted more than 18 weeks;

urogynecological conditions (incontinence, pelvic organ prolapse $>1$ stage at the pelvic organ prolapse quantification [33]);

active urinary or vaginal infection (or earlier in last 3 months);

$-$

use of medication that can affect pain perception (analgesics, opiates, antiepileptic, muscle relaxant);

$-$

anterior vulvar or vaginal surgery;

post-menopausal status;

major psychological conditions (i.e. depressive symptoms, anxiety) that could present a risk for patient safety;

refusal to refrain from treatments until the 6-month follow-up assessment;

$-$

previously received physiotherapy treatment or overnight use of lidocaine; and

$-$

any coexisting significant medical condition likely to interfere with study procedures (e.g. cardiovascular, hematological, central nervous system, pulmonary, renal).

\subsection{Baseline assessment}

Interested women were invited to contact the research coordinator for a detailed explanation of the study followed by a pre-screening of the eligibility criteria. Participants then took part in a medical examination with a gynecologist of our team to confirm the diagnosis of PVD. Women were then convened to the first evaluation performed by a trained physiotherapist. After signing the informed consent, the women underwent a standardized examination: 1) a structured interview for gathering socio-demographic information, pain, medical and gynecological history; 2) validated questionnaires for evaluating pain, sexual function and psychological variables and 3) physical examination (including PFM morphometry and function). An assessment schedule is shown in Table 2. 
Table 2.

Assessment schedule.

\begin{tabular}{|c|c|c|c|c|}
\hline & $\begin{array}{l}\text { Baseline } \\
\text { assessment } \\
\text { by a physio- } \\
\text { therapist }\end{array}$ & $\begin{array}{l}10 \text {-week } \\
\text { treatment }\end{array}$ & $\begin{array}{l}\text { Post-treatment } \\
\text { assessment } \\
\text { ( } 2 \text { weeks after } \\
\text { treatment) }\end{array}$ & $\begin{array}{l}\text { Follow-up } \\
\text { assessment } \\
\text { (6 months after } \\
\text { treatment) }\end{array}$ \\
\hline \multirow{2}{*}{$\begin{array}{l}\text { Contact information } \\
\text { Informed consent }\end{array}$} & $X$ & & $\mathrm{X}$ update & X update \\
\hline & $\mathrm{x}$ & & & \\
\hline $\begin{array}{l}\text { Structured interview } \\
\text { (socio-demographic } \\
\text { information, pain and } \\
\text { gynecological } \\
\text { history) }\end{array}$ & $\mathrm{X}$ & & X update & X update \\
\hline Questionnaires & $X$ & & $x$ & $\mathrm{X}$ \\
\hline $\begin{array}{l}\text { Physical } \\
\text { examination: }\end{array}$ & $X$ & & $x$ & $x$ \\
\hline $\begin{array}{l}\text {-PFM morphology } \\
\text { (transperineal } \\
\text { ultrasound) }\end{array}$ & & & & \\
\hline $\begin{array}{l}\text {-PFM function } \\
\text { (dynamometry) }\end{array}$ & & & & \\
\hline $\begin{array}{l}\text { Intervention } \\
\text { (physiotherapy or } \\
\text { lidocaine) }\end{array}$ & & $x$ & & \\
\hline $\begin{array}{lr}\text { Diary } & \text { reporting } \\
\text { adherence } & \text { to } \\
\text { treatment } & \end{array}$ & & $x$ & & \\
\hline
\end{tabular}

PFM: Pelvic floor muscles.

\subsection{Randomization and blinding}

Eligible participants were assigned with equal probability to one of the two treatment groups. Participant randomization was stratified by center, using random permuted blocks of 4 and 6 using a list computer-generated managed by an independent individual. Investigators, data analysts, gynecologists and physiotherapists in charge of the outcome evaluations remain blinded to the trial group allocation. The treatment was explained by the research coordinator at each site after the baseline evaluation and patients were reminded not to reveal their treatment in the initial consent form as well as at the beginning of each evaluation.

\subsection{Post-treatment and follow-up assessments}

Post-treatment evaluation occurs two weeks after treatment as well as at 6-months follow-up, when the same procedures as in the baseline evaluation are repeated. Further, the participants are asked to rate the perceived improvement and to report any adverse effects related to treatment. 


\subsection{Outcomes}

Primary and secondary outcomes are presented detailed in Table 3. The primary-outcome measure is the NRS, which is used to quantify pain during intercourse. The participant is asked to evaluate the average intensity of pain on an 11-point scale from 0 (no pain) to 10 (most intense pain). This scale, highly recommended by the Initiative on Methods, Measurement, and Pain Assessment in Clinical Trials (IMMPACT) [34], is widely used in RCTs for chronic pain conditions as well as for women with PVD and has shown good validity, reliability and responsiveness [18], [24], [30] and [34].

Table 3.

Outcome measures.

\begin{tabular}{|c|c|}
\hline \multicolumn{2}{|l|}{ Primary outcome measure } \\
\hline Pain intensity (NRS) ${ }^{\mathrm{a}}[34]$ & $\begin{array}{l}\text { Average pain intensity reported during sexual } \\
\text { intercourse }\end{array}$ \\
\hline \multicolumn{2}{|l|}{ Secondary outcome measures } \\
\hline $\mathrm{MPQ}^{\mathrm{b}}[35]$ & $\begin{array}{l}\text { Quality of pain: sensory, affective and evaluative } \\
\text { components of pain }\end{array}$ \\
\hline $\mathrm{FSFI}^{\mathrm{C}}[36]$ & $\begin{array}{l}\text { Sexual functioning: desire and arousal, lubrication, } \\
\text { orgasm, satisfaction and pain/discomfort }\end{array}$ \\
\hline IEMSS $^{\mathrm{d}}[37]$ & Sexual satisfaction \\
\hline $\operatorname{FSDS}^{\mathrm{e}}[38]$ & Sexually related personal distress \\
\hline PCS-CF ${ }^{\dagger}[39]$ & $\begin{array}{l}\text { Pain catastrophizing: rumination, magnification and } \\
\text { helplessness towards pain }\end{array}$ \\
\hline $\mathrm{STAl}^{\mathrm{g}}[40]$ & $\begin{array}{l}\text { Anxiety as a personality trait and in response to a } \\
\text { situation }\end{array}$ \\
\hline$B D I-I^{h}[41]$ & Depressive symptoms \\
\hline PASS-20'[42] and [55] & Fear of pain \\
\hline PGIC $[43]$ & Patient's perceived changes after treatment \\
\hline 4D ultrasound [12] & PFM morphology \\
\hline Dynamometry [47], [48], [49], [50],[51], [52], [53] and [54] & PFM function \\
\hline
\end{tabular}

PFM: Pelvic floor muscles.

a NRS: Numerical Rating Scale.

b MPQ: McGill-Melzack Pain Questionnaire.

c FSFI: Female Sexual Function Index.

d IEMSS: Interpersonal Exchange Model of Sexual Satisfaction Questionnaire.

e FSDS: Female Sexual Distress Scale.

f PCS-CF: Pain Catastrophizing Scale.

g STAI: State and Trait Anxiety Inventory.

h BDI-II: Beck Depression Inventory.

i PASS-20: Pain Anxiety Symptoms Scale.

j PGIC: Patient's Global Impression of Change. 
Several secondary outcomes are assessed in this study, in line with the IMMPACT consensus recommending a multi-facetted evaluation of pain, and also an evaluation of the impact of pain on function [34]. For each outcome we have selected measure instruments that have excellent validity, reliability and responsiveness to change. The McGill-Melzack pain questionnaire (MPQ) assesses the quality of pain by investigating its sensory, affective and evaluative components [35].

The Female Sexual Function Index (FSFI) is a multidimensional measure of sexual function evaluating desire, arousal, lubrication, orgasm, satisfaction and pain [36]. The Interpersonal Exchange Model of Sexual Satisfaction Questionnaire (IEMSS) is used to investigate sexual satisfaction [37]. Lastly, the Female Sexual Distress Scale (FSDS) 12-item questionnaire is employed to measure sexually-related personal distress [38].

Several psychological variables are also evaluated: (1) the Pain Catastrophizing Scale (PCS), a 13item questionnaire which evaluates pain-related rumination, helplessness and magnification [39]; (2) Spielberger's State-Trait Anxiety Inventory (STAI), which allows a distinction to be made between anxiety as a trait of personality or an emotional response to a situation [40]; (3) the Beck Depression Inventory (BDI-II), a 21-item questionnaire assessing symptoms of depression [41]; (4) the Pain Anxiety Symptoms Scale (PASS-20), evaluating fear of pain during intercourse [42]; and (5) the Patient's Global Impression of Change (PGIC), a patient-reported measure selected to evaluate perceived reduction in pain using a 7-point scale that ranges from 'very much improved' to 'very much worse' [43].

Alterations in PFM morphology have been demonstrated in women with PVD using transperineal four-dimensional (4D) ultrasound [12]. Using this validated and reliable methodology [44], [45] and [46], several parameters are evaluated at rest and during PFM contraction: levator hiatus area and diameter, bladder neck positioning and displacement as well as levator plate and anorectal angles. An intravaginal dynamometric speculum, developed by our team to evaluate PFM function [47], [48], [49] and [50], is also used in this trial. This instrument has been widely assessed for its psychometric properties including its reliability, validity and responsiveness [49], [50], [51], [52], [53] and [54]. PFM dysfunctions identified in women with PVD, namely heightened passive forces, stiffness and lower strength, speed of contraction and endurance [11], are evaluated in this trial.

\subsection{Multimodal physiotherapy treatment}

The physiotherapy treatment consists of ten 60-minute weekly sessions with a physiotherapist specialized in gynecological pain. With a view to investigating an intervention that reflects clinical practice, different treatment modalities were selected according to the usual practice of physiotherapists in North America [14]. The educational component of our intervention includes explanations about the PVD pathophysiology, the involvement of PFM, healthy vulvo-vaginal and sexual behaviors, chronic pain and factors influencing pain intensity, relaxation techniques, sexual function and recovery of non-painful sexual activities. Approximately 20-25 min are 
dedicated to manual techniques (stretching, myofascial release techniques, pressure and massage) to increase flexibility, release muscle tensions and trigger points in the PFMs. Depending on the patient's pain referral pattern, these techniques are also applied to the obturator internus, piriformis, gluteus, adductors and abdominal muscles. The techniques are adapted to each patient and evolve throughout the treatment sessions (e.g. from one to two fingers, more pressure or stretching applied). A 20 min period of biofeedback (Evadri, ${ }^{\circledR}$ Hollister, Biomation, Canada) is applied using a small intravaginal probe to promote relaxation, control and strength, speed of contraction and endurance. The daily home exercise program incorporates deep breathing exercises, PFM contraction and control, stretching using dilatator as well as massage and tissue mobilization of the vulvar vestibule. Additionally, partners are convened to attend a session to discuss the pathophysiology of PVD, sexual activities and how to perform PFM stretching exercises.

\subsection{Lidocaine treatment}

For the lidocaine treatment, participants are asked to apply the $5 \%$ lidocaine ointment $(50 \mathrm{mg} / \mathrm{g}$, Lidocan $^{\circledast}$, Odan Lab, $35 \mathrm{~g}$ ) to the vestibule every night as described by Zolnoun et al. [26]. After gently cleaning and drying the vulvar area, the participants are asked to apply a small blob of ointment (size of a marble) on the affected region (i.e. the vestibule) without rubbing. The same amount is then applied on a small cotton gauze pad which is positioned at the entry of the vagina. The gauze should remain in continuous contact with the vestibule overnight (approximately $8 \mathrm{~h}$ ). The participant is asked to wear cotton underwear to help maintain the gauze in place. The treatment is repeated nightly for 10 weeks. The procedure is explained verbally by the research coordinator and an instruction sheet is given.

\subsection{Sample size}

The sample size was calculated based on the primary outcome - the intensity of pain during sexual intercourse evaluated with the NRS. Statistical power analysis examined the requirements to detect a clinically significant difference of 1.5 points between the two treatments with a two-sided significance level of $0.05,80 \%$ power and 3.47 standard deviation [26]. Several studies of musculoskeletal conditions indicated that a reduction of 1.5 to 2.0 points in pain corresponds to a minimal clinically significant difference [56] and [57]. Moreover, the IMMPACT group recommends a $30 \%$ pain reduction as a threshold for clinical significance [34]; this would correspond to 1.8 points based on our previous study (baseline average pain of 6/10) [12]. In our studies investigating different treatments, a difference of 1.5 to 4.67 points was observed [18] and [24]. In the most conservative scenario, a sample of 170 participants (85 per group) would be necessary. To account for an expected drop-out rate of $20 \%$ after 6 months [18] and [28], 212 participants (170/0.80) need to be enrolled at baseline. This number provides sufficient power to meet the secondary objectives with power ranging from 84.4-99.9\%.

\subsection{Statistical analysis plan}


Primary analysis: Analyses will be done based on the intention-to-treat principle. A multilevel model of change (growth model) will evaluate and compare the efficacy of the interventions by comparing values for the pain during intercourse at the different time-points and between groups [58]. This type of analysis takes into account the dependency between repeated measures without requiring identical intervals between time-points. It also allows the use of partial data from women who did not participate in all measurements. A piecewise lineargrowth model will be estimated since we anticipate that the changes between the evaluations pre- and post-treatments will be greater than with the 6-month follow-up. Moreover, the statistical model will be adjusted for the stratification variable (i.e. center).

Secondary analysis: The same analysis will be used to compare the efficacy of the treatments on the secondary variables. The number of tests will be taken into account as the level of significance will be lowered using the Bonferroni correction. Exploratory analysis will also be used to determine the effect of adherence to the procedures on the treatment efficacy.

\section{Current status of the trial}

The first participant was enrolled on May 11, 2012. As shown in Fig. 1, a total of 537 women reporting pain at the entry of the vagina during intercourse were interested in participating and 223 were found eligible at the telephone pre-screening process. Most common reasons for exclusion included having previously received physiotherapy or overnight lidocaine (21\%), previous pregnancies (18\%), pelvic and urogynecological pathologies (18\%) and age (11\%). As of August 30, 2015, the total sample size of 212 women had been recruited and randomized. Assessments are ongoing. It is expected that the study will be completed by December 2015 with the last 6-month follow-up assessment.

\section{Discussion}

In the development and implementation of this first RCT investigating the efficacy of multimodal physiotherapy in women with PVD, several methodological issues were considered including the choice of intervention, the eligibility criteria, the duration of interventions, the participant adherence as well as other methods to control for potential bias. Moreover, when the study was launched, some challenges pertaining to low rate of recruitment and retention issues were encountered and some additional strategies were developed and implemented.

\subsection{Choice of interventions}

This trial compares physiotherapy, a promising intervention judged to be effective, with topical overnight lidocaine application, a frequently used first-line intervention [16] and [17]. Physiotherapy modalities, taken separately, have been shown to produce a significant reduction in pain during intercourse in women with PVD [18], [19] and [20]. However, their efficacy seems to be lower compared to a multimodal intervention [24] and [25]. Recommended in the treatment of chronic pain [59], a multimodal approach was selected to better represent treatment delivery in a clinical setting based on a survey of physiotherapists [14]. Despite 
excellent preliminary results [24] and [25], multimodal physiotherapy has never been studied in a randomized clinical trial with a large sample. With regard to the topical application of lidocaine, we also intended to reflect current clinical practice. Overnight application of lidocaine $5 \%$ ointment was selected in our trial as this protocol has shown the highest efficacy so far [26] compared to daily application of lidocaine [27] and [28]. Application of lidocaine before intercourse has been described in clinical guidelines [16] and [17], but no data are yet available to support its efficacy. Furthermore, the design of this study does not entail comparison with an untreated or placebo control group. It was considered unethical to withhold women from treatment after previous studies had already reported effective treatments for PVD. Moreover, as patients participate actively in treatment, sham multimodal physiotherapy seemed unlikely to be credible for women. Our choice of study design is in line with current strategies used in nonpharmaceutical trials whereas an active control or usual care comparison group is generally selected in order to prevent attrition bias due to an untreated study arm or non-credible placebo treatment [60]. Consequently, we opted for comparing multimodal physiotherapy to one of the most prescribed first-line treatments [15].

\subsection{Eligibility criteria}

The targeted sample included women 18 to 45 years old. As shown by Harlow et al. [61], this age group is generalizable to the population of women with PVD given that it covers $87 \%$ of affected women. This is also consistent with our findings as only $11 \%$ of women interested in participating were excluded because of age. Inclusion of women close to the age of menopause might have introduced other pathologies related to dyspareunia such as vulvo-vaginal atrophy. Likewise, to avoid pain explained by childbirth-related lesions and to limit the confounding effect of pregnancy and delivery on PFM function, only nulliparous women were recruited. Women with moderate or severe pain during intercourse were included as they are representative of the PVD population [30], and their pain may also correspond to a typical level of severity at which many women will seek medical advice [6]. It will also prevent a floor effect and thus ensure that the intervention will have a measurable efficacy [62]. Moreover, a 6-month duration of symptoms was selected based on the DSM-V criteria for genito-pelvic pain/penetration disorders [63]. Having a stable sexual partner was one of the inclusion criteria. Although this may affect external validity, as not all women with PVD are currently engaged in a relationship, this criterion was necessary to investigate the study's primary outcome, i.e. change in pain intensity during intercourse. It has been suggested that oral contraceptives could influence vestibular sensitivity [64] and PVD development [9] but conflicting results were found in large epidemiological studies showing a non-significant impact [65] and [66]. It was therefore decided not to exclude women taking oral contraceptives but to verify this potential confounder and if relevant, incorporate it as a covariate in the statistical model. In sum, the eligibility criteria were chosen to best represent the reality of women with PVD while reducing the possibility of bias arising from a wrong diagnosis or confounding factors.

\subsection{Duration of interventions}


Patients were randomly assigned to one of the two 10-week treatment groups. The use of weekly physiotherapy treatments over a period of 10 weeks corresponds to current clinical practice [14]. In fact, to achieve the treatment's objective to target PFM function, an 8- to 12week training period is generally recommended [67]. As for lidocaine application, previous studies report treatment periods ranging from 7 weeks of an overnight application [26] to 12 weeks of a 4-7 times daily application [27] and [28]. Since the overnight application was chosen in this study, a 10-week treatment is in accordance with previous studies and allows uniformity in the length of the two treatments. In order to limit the potential bias associated with the therapeutic relationship created with the physiotherapist, a weekly follow-up of the women in the lidocaine application group was included. This 15-minute follow-up phone call with the study coordinator allows the patient to ask questions during the treatments, address her worries and promote adherence to treatment.

\subsection{Outcome measures}

In this study, outcome measures were selected to evaluate every dimension of PVD and the potential impact of the treatment. The assessment tools used in this trial adhere to the recommendations of the IMMPACT consensus [34]. Accordingly, this protocol proposes a thorough evaluation of pain intensity and characteristics, physical functioning (sexual and muscle function in this study), emotional functioning and participant ratings of global improvement [34]. The primary outcome (i.e. pain intensity during intercourse) was selected based on its clinical relevance for women with PVD and, also, because the majority of current studies have also included this measure, which will facilitate inter-trial comparisons. All tools used in this study have good psychometric properties and have been used in our previous studies.

\subsection{Participant adherence to treatment}

First, at recruitment, a motivational interview is held with each participant and the barriers to following study procedures are discussed to promote adherence [68]. The participants are also asked to keep a diary to indicate if they do their exercises (physiotherapy group) or apply the ointment (lidocaine group) throughout the 10 weeks. Participants also indicate whether they feel pain related to the treatment and during intercourse. The diary is reviewed weekly by the physiotherapist (physiotherapy treatment) or study coordinator (lidocaine treatment) to discuss the patient's adherence to treatment and evolution of her pain. Moreover, several time periods for physiotherapy and telephone calls are proposed to the participant to accommodate her schedule.

\subsection{Methods to control bias}

Physiotherapists at both sites followed a standardized training and were given a written detailed evaluation form and a treatment protocol ensuring consistency of treatment and assessment procedure as well as reducing bias due to the evaluators. The assessment and treatment files are reviewed on a regular basis by the principal investigator in order to identify and address 
protocol deviations. In order to keep the blinding, treatment physiotherapists never conduct pre- and post-treatment or 6-month follow-up assessments. Lastly, women are also required to abstain from any other treatment during the study period (until the 6-month follow-up). To evaluate whether contamination of the intervention occurred, participants are asked at the post-treatment and 6-month assessments to declare if they received any other treatments than the one assigned to them during the study period. Participants are reminded of their appointments and receive a $\$ 40$ compensation for each evaluation in order to enhance the likelihood of complete follow-up.

\subsection{Lessons learned}

Concerns about low recruitment rate were raised when the study was launched. Whereas initial recruitment sources mostly relied on posters and physician referrals, multiple recruitment strategies were thereafter deployed and intensified to increase the recruitment rate. The addition of newspaper ads, Facebook and web initiatives allowed us to increase the recruitment rate from 2 patients per month in the first year to more than 7 patients per month. Given the age group of the targeted sample, it is interesting to notice that Facebook and web initiatives represent $30 \%$ of the recruitment sources. The other recruitment source are distributed as follow: Posters in clinics, universities, professional schools, restaurants, gyms $32 \%$, references by health professionals $17 \%$, ads in local newspapers $11 \%$, word to mouth $8 \%$ and public conference $2 \%$. Furthermore, challenges in reaching the participants for their 6 month follow-up were encountered with the first participants completing the trial which was explained by the fact that a portion of participants moved to the cosmopolitan area for their education. Even though we excluded women unable to engage in the study until the last follow-up assessment, this population was more prone to moving during their studies and leaving the area when graduating. We have implemented several strategies to prevent attrition: 1) Collecting multiple contact information and reviewing these at each assessment (phone numbers, email); 2) adding a phone call at 3 months post-treatment to maintain contact with the participant and plan the follow-up assessment and 3) emphasizing the value of study completion and appreciation for patient's contribution at each assessment and at the 3 month post-treatment phone call.

\section{Conclusion}

This bi-center RCT addresses an important need to provide scientific evidence in support of PVD treatment option, a frequent yet neglected problem with significant psychosocial repercussions. It is the first RCT investigating the efficacy of a multimodal physiotherapy compared to another

first-line treatment, overnight lidocaine application. Findings from this trial will provide important guidance for health professionals about the efficacy of two first-line treatments and will help to better direct their patients towards the best empirically validated treatment.

\section{Conflict of interest statement}

The authors declare that they have neither competing interests nor conflict of interest. 


\section{Ethics}

Ethics approval for this study was obtained from the Research Ethics Committees of the Centre hospitalier universitaire de Sherbrooke and Institut Universitaire de gériatrie de Montréal.

\section{Acknowledgments}

This study is supported by a grant from the Canadian Institute of Health Research (MOP115028). Dr. Mélanie Morin is supported by a Junior 1 research scholar of the Fonds de la recherche du Québec-Santé. The authors wish to acknowledge the collaboration of the physiotherapists doing the evaluations and treatments in this project.

\section{References}

[1] B.L. Harlow, C.G. Kunitz, R.H. Nguyen, S.A. Rydell, R.M. Turner, R.F. MacLehose

Prevalence of symptoms consistent with a diagnosis of vulvodynia: population-based estimates from 2 geographic regions

Am. J. Obstet. Gynecol., 210 (1) (2014), p. 40 (e41-48)

[2] E.G. Friedrich Jr.

Vulvar vestibulitis syndrome

J. Reprod. Med., 32 (2) (1987), pp. 110-114

[3] K.A. Payne, Y.M. Binik, R. Amsel, S. Khalife

When sex hurts, anxiety and fear orient attention towards pain

Eur. J. Pain, 9 (4) (2005), pp. 427-436

[4] L.D. Arnold, G.A. Bachmann, R. Rosen, S. Kelly, G.G. Rhoads

Vulvodynia: characteristics and associations with comorbidities and quality of life

Obstet. Gynecol., 107 (3) (2006), pp. 617-624

[5] G. Desrochers, S. Bergeron, T. Landry, M. Jodoin

Do psychosexual factors play a role in the etiology of provoked vestibulodynia? A critical review J. Sex Marital Ther., 34 (3) (2008), pp. 198-226

[6] A.S. Gordon, M. Panahian-Jand, F. McComb, C. Melegari, S. Sharp

Characteristics of women with vulvar pain disorders: responses to a web-based survey

J. Sex Marital Ther., 29 (Suppl. 1) (2003), pp. 45-58

[7] G.A. Bachmann, R. Rosen, L.D. Arnold, I. Burd, G.G. Rhoads, S.R. Leiblum, N. Avis

Chronic vulvar and other gynecologic pain: prevalence and characteristics in a self-reported survey

J. Reprod. Med., 51 (1) (2006), pp. 3-9 
[8] S.S. Witkin, S. Gerber, W.J. Ledger

Influence of interleukin-1 receptor antagonist gene polymorphism on disease

Clin. Infect. Dis., 34 (2) (2002), pp. 204-209

[9] A.T. Goldstein, Z.R. Belkin, J.M. Krapf, W. Song, M. Khera, S.L. Jutrzonka, N.N. Kim, L.J. Burrows, I. Goldstein

Polymorphisms of the androgen receptor gene and hormonal contraceptive induced provoked vestibulodynia

J. Sex. Med., 11 (11) (2014), pp. 2764-2771

Article | PDF (365 K) | Full Text via CrossRef | View Record in Scopus | Citing articles (11)

[10]

N. Bohm-Starke, M. Hilliges, G. Brodda-Jansen, E. Rylander, E. Torebjork

Psychophysical evidence of nociceptor sensitization in vulvar vestibulitis syndrome

Pain, 94 (2) (2001), pp. 177-183

[11] M. Morin, S. Bergeron, S. Khalifé, I. Binik, S. Ouellet

Dynamometric assessment of the pelvic floor muscle function in women with and without provoked vestibulodynia

Neurourol. Urodyn., 29 (2010), pp. 1140-1141

[12] M. Morin, S. Bergeron, S. Khalife, M.H. Mayrand, Y.M. Binik

Morphometry of the pelvic floor muscles in women with and without provoked vestibulodynia using 4D ultrasound

J. Sex. Med., 11 (3) (2014), pp. 776-785

[13] E. Gentilcore-Saulnier, L. McLean, C. Goldfinger, C.F. Pukall, S. Chamberlain

Pelvic floor muscle assessment outcomes in women with and without provoked vestibulodynia and the impact of a physical therapy program

J. Sex. Med., 7 (2010), pp. 1003-1022

[14] D. Hartmann, M.J. Strauhal, C.A. Nelson

Treatment of women in the United States with localized, provoked vulvodynia: practice survey of women's health physical therapists

J. Reprod. Med., 52 (1) (2007), pp. 48-52

[15] B.D. Reed, H.K. Haefner, L. Edwards

A survey on diagnosis and treatment of vulvodynia among vulvodynia researchers and members of the International Society for the Study of Vulvovaginal Disease

J. Reprod. Med., 53 (12) (2008), pp. 921-929 
[16] ACOG

Committee opinion: number 345, October 2006: vulvodynia

Obstet. Gynecol., 108 (4) (2006), pp. 1049-1052

[17] D. Mandal, D. Nunns, M. Byrne, J. McLelland, R. Rani, J. Cullimore, D. Bansal, F. Brackenbury, G. Kirtschig, M. Wier

Guidelines for the management of vulvodynia

Br. J. Dermatol., 162 (6) (2010), pp. 1180-1185

[18] S. Bergeron, Y.M. Binik, S. Khalife, K. Pagidas, H.I. Glazer, M. Meana, R. Amsel

A randomized comparison of group cognitive-behavioral therapy, surface electromyographic biofeedback, and vestibulectomy in the treatment of dyspareunia resulting from vulvar vestibulitis

Pain, 91 (3) (2001), pp. 297-306

[19] M.P. Fitzgerald, R. Kotarinos

Rehabilitation of the short pelvic floor. II: treatment of the patient with the short pelvic floor Int. Urogynecol. J. Pelvic Floor Dysfunct., 14 (4) (2003), pp. 269-275 discussion 275

[20] T.O. Idama, D.W. Pring

Vaginal dilator therapy-an outpatient gynaecological option in the management of dyspareunia J. Obstet. Gynaecol., 20 (3) (2000), pp. 303-305

[21] P. Downey, I. Frederick

Physical therapy treatment for vulvar vestibulitis: a case report

J. Womens Health Phys. Therap., 30 (1) (2006), pp. 16-19

[22] K.A. Fisher

Management of dyspareunia and associated levator ani muscle overactivity

Phys. Ther., 87 (7) (2007), pp. 935-941

[23] A. Holland

Case report: physical therapy intervention for dyspareunia

J. Sect. Women's Health, 27 (1) (2003), pp. 18-20

[24] S. Bergeron, C. Brown, M.J. Lord, M. Oala, Y.M. Binik, S. Khalife

Physical therapy for vulvar vestibulitis syndrome: a retrospective study

J. Sex Marital Ther., 28 (3) (2002), pp. 183-192 
[25] C. Goldfinger, C.F. Pukall, E. Gentilcore-Saulnier, L. McLean, S. Chamberlain

A prospective study of pelvic floor physical therapy: pain and psychosexual outcomes in provoked vestibulodynia

J. Sex. Med., 6 (7) (2009), pp. 1955-1968

[26] D.A. Zolnoun, K.E. Hartmann, J.F. Steege

Overnight $5 \%$ lidocaine ointment for treatment of vulvar vestibulitis

Obstet. Gynecol., 102 (1) (2003), pp. 84-87

[27] I. Danielsson, T. Torstensson, G. Brodda-Jansen, N. Bohm-Starke

EMG biofeedback versus topical lidocaine gel: a randomized study for the treatment of women with vulvar vestibulitis

Acta Obstet. Gynecol. Scand., 85 (11) (2006), pp. 1360-1367

[28] D.C. Foster, M.B. Kotok, L.S. Huang, A. Watts, D. Oakes, F.M. Howard, E.L. Poleshuck, C.J. Stodgell, R.H. Dworkin

Oral desipramine and topical lidocaine for vulvodynia: a randomized controlled trial

Obstet. Gynecol., 116 (3) (2010), pp. 583-593

[29] J.A. Turner, G. Franklin, P.J. Heagerty, R. Wu, K. Egan, D. Fulton-Kehoe, J.V. Gluck, T.M. Wickizer

The association between pain and disability

Pain, 112 (3) (2004), pp. 307-314

[30] S. Bergeron, Y.M. Binik, S. Khalifé, K. Pagidas, H.I. Glazer

Vulvar vestibulitis syndrome: reliability of diagnosis and evaluation of current diagnostic criteria Obstet. Gynecol., 98 (1) (2001), pp. 45-51

[31] M. Moyal-Barracco, P.J. Lynch

2003 ISSVD terminology and classification of vulvodynia: a historical perspective

J. Reprod. Med., 49 (10) (2004), pp. 772-777

[32] American Psychiatric Association

Sexual pain disorders

Diagnostic and Statistical Manual of Mental Disorders : DSM-IV-TR, American Psychiatric Association, Washington, DC (2000), pp. 554-558

[33] R.C. Bump, A. Mattiasson, K. Bo, L.P. Brubaker, J.O. DeLancey, P. Klarskov, B.L. Shull, A.R. Smith

The standardization of terminology of female pelvic organ prolapse and pelvic floor dysfunction Am. J. Obstet. Gynecol., 175 (1) (1996), pp. 10-17 
[34] R.H. Dworkin, D.C. Turk, J.T. Farrar, J.A. Haythornthwaite, M.P. Jensen, N.P. Katz, R.D. Kerns, G. Stucki, R.R. Allen, N. Bellamy, D.B. Carr, J. Chandler, P. Cowan, R. Dionne, B.S. Galer, S. Hertz, A.R. Jadad, L.D. Kramer, D.C. Manning, S. Martin, C.G. McCormick, M.P. McDermott, P. McGrath, S. Quessy, B.A. Rappaport, W. Robbins, J.P. Robinson, M. Rothman, M.A. Royal, L. Simon, J.W. Stauffer, W. Stein, J. Tollett, J. Wernicke, J. Witter

Core outcome measures for chronic pain clinical trials: IMMPACT recommendations

Pain, 113 (1-2) (2005), pp. 9-19

[35] R. Melzack

The McGill Pain Questionnaire: major properties and scoring methods

Pain, 1 (3) (1975), pp. 277-299

[36] R. Rosen, C. Brown, J. Heiman, S. Leiblum, C. Meston, R. Shabsigh, D. Ferguson, R. D'Agostino Jr.

The Female Sexual Function Index (FSFI): a multidimensional self-report instrument for the assessment of female sexual function

J. Sex Marital Ther., 26 (2) (2000), pp. 191-208

[37] K. Lawrance, E.S. Byers

Sexual satisfaction in long-term heterosexual relationships: the interpersonal exchange model of sexual satisfaction

Pers. Relat., 2 (4) (1995), pp. 267-285

[38] L.R. Derogatis, R. Rosen, S. Leiblum, A. Burnett, J. Heiman

The Female Sexual Distress Scale (FSDS): initial validation of a standardized scale for assessment of sexually related personal distress in women

J. Sex Marital Ther., 28 (4) (2002), pp. 317-330

[39] M.J.L. Sullivan, S.R. Bishop, J. Pivik

The Pain Catastrophizing Scale: development and validation

Psychol. Assess., 7 (1995), pp. 524-532

[40] C.D. Spielberger, R. Gorsuch, R. Lushene

The State-Trait Anxiety Inventory (STAI) Manual

Consulting Psychologists Press, Palo Alto, CA (1970)

[41] C.A. Harris, J.L. D'Eon

Psychometric properties of the Beck Depression Inventory-second edition (BDI-II) in individuals with chronic pain

Pain, 137 (3) (2008), pp. 609-622 
[42] L.M. McCracken, L. Dhingra

A short version of the Pain Anxiety Symptoms Scale (PASS-20): preliminary development and validity

Pain Res. Manag., 7 (1) (2002), pp. 45-50

[43] J.T. Farrar, J.P. Young Jr., L. LaMoreaux, J.L. Werth, R.M. Poole

Clinical importance of changes in chronic pain intensity measured on an 11-point numerical pain rating scale

Pain, 94 (2) (2001), pp. 149-158

[44] M. Majida, I.H. Braekken, W. Umek, K. Bo, J. Saltyte Benth, E.M. Ellstrom

Interobserver repeatability of three- and four-dimensional transperineal ultrasound assessment of pelvic floor muscle anatomy and function

Ultrasound Obstet. Gynecol., 33 (5) (2009), pp. 567-573

[45] I.H. Braekken, M. Majida, M.E. Engh, K. Bo

Test-retest reliability of pelvic floor muscle contraction measured by $4 \mathrm{D}$ ultrasound Neurourol. Urodyn., 28 (1) (2009), pp. 68-73

[46] M.M. Weinstein, S.A. Jung, D.H. Pretorius, C.W. Nager, D.J. den Boer, R.K. Mittal The reliability of puborectalis muscle measurements with 3-dimensional ultrasound imaging Am. J. Obstet. Gynecol., 197 (1) (2007), p. 68 (e61-66)

[47] C. Dumoulin, D. Bourbonnais, M.C. Lemieux

Development of a dynamometer for measuring the isometric force of the pelvic floor musculature

Neurourol. Urodyn., 22 (7) (2003), pp. 648-653

[48] M. Morin, D. Gravel, D. Bourbonnais, C. Dumoulin, S. Ouellet, J.F. Pilon

Application of a new method in the study of pelvic floor muscle passive properties in continent women

J. Electromyogr. Kinesiol., 20 (5) (2010), pp. 795-803

[49] M. Morin, D. Gravel, D. Bourbonnais, C. Dumoulin, S. Ouellet

Reliability of dynamometric passive properties of the pelvic floor muscles in postmenopausal women with stress urinary incontinence

Neurourol. Urodyn., 27 (8) (2008), pp. 819-825

[50] M. Morin, C. Dumoulin, D. Gravel, D. Bourbonnais, M.C. Lemieux

Reliability of speed of contraction and endurance dynamometric measurements of the pelvic floor musculature in stress incontinent parous women Neurourol. Urodyn., 26 (3) (2007), pp. 397-403 discussion 404 
[51] C. Dumoulin, D. Gravel, D. Bourbonnais, M.C. Lemieux, M. Morin

Reliability of dynamometric measurements of the pelvic floor musculature

Neurourol. Urodyn., 23 (2) (2004), pp. 134-142

[52] M. Morin, C. Dumoulin, D. Bourbonnais, D. Gravel, M.C. Lemieux

Pelvic floor maximal strength using vaginal digital assessment compared to dynamometric measurements

Neurourol. Urodyn., 23 (4) (2004), pp. 336-341

[53] M. Morin, D. Gravel, S. Ouellet, C. Dumoulin, D. Bourbonnais

Influence of intra-abdominal pressure on the validity of pelvic floor dynamometric measurements

Neurourol. Urodyn., 25 (6) (2006), pp. 530-531

[54] C. Dumoulin, D. Bourbonnais, M. Morin, D. Gravel, M.C. Lemieux

Predictors of success for physiotherapy treatment in women with persistent postpartum stress urinary incontinence

Arch. Phys. Med. Rehabil., 91 (7) (2011), pp. 1059-1063

[55] M.J. Coons, H.D. Hadjistavropoulos, G.J. Asmundson

Factor structure and psychometric properties of the Pain Anxiety Symptoms Scale-20 in a community physiotherapy clinic sample

Eur. J. Pain, 8 (6) (2004), pp. 511-516

[56] F.M. Kovacs, V. Abraira, A. Royuela, J. Corcoll, L. Alegre, M. Tomas, M.A. Mir, A. Cano, A. Muriel, J. Zamora, M.T. Del Real, M. Gestoso, N. Mufraggi

Minimum detectable and minimal clinically important changes for pain in patients with nonspecific neck pain

BMC Musculoskelet. Disord., 9 (2008), p. 43

[57] R.W. Ostelo, R.A. Deyo, P. Stratford, G. Waddell, P. Croft, M. Von Korff, L.M. Bouter, H.C. de Vet Interpreting change scores for pain and functional status in low back pain: towards international consensus regarding minimal important change

Spine (Phila Pa 1976), 33 (1) (2008), pp. 90-94

[58] Singer JD, Willett JB editors |. Title |, Vol. Volume |. City |: Publisher |, Year |.

[59] D. Cawley, M.I. Bennett

Management of pain

Br. J. Hosp. Med. (Lond.), 70 (4) (2009), pp. 197-201 
[60] I. Boutron, F. Tubach, B. Giraudeau, P. Ravaud

Methodological differences in clinical trials evaluating nonpharmacological and pharmacological treatments of hip and knee osteoarthritis

JAMA, 290 (8) (2003), pp. 1062-1070

[61] B.L. Harlow, E.G. Stewart

A population-based assessment of chronic unexplained vulvar pain: have we underestimated the prevalence of vulvodynia?

J. Am. Med. Wom. Assoc., 58 (2) (2003), pp. 82-88

[62] R.H. Dworkin, D.C. Turk, S. Peirce-Sandner, R. Baron, N. Bellamy, L.B. Burke, A. Chappell, K. Chartier, C.S. Cleeland, A. Costello, P. Cowan, R. Dimitrova, S. Ellenberg, J.T. Farrar, J.A. French, I. Gilron, S. Hertz, A.R. Jadad, G.W. Jay, J. Kalliomaki, N.P. Katz, R.D. Kerns, D.C. Manning, M.D. MP, M.G. PJ, A. Narayana, L. Porter, S. Quessy, B.A. Rappaport, C. Rauschkolb, B.B. Reeve, T. Rhodes, C. Sampaio, D.M. Simpson, J.W. Stauffer, G. Stucki, J. Tobias, R.E. White, J. Witter

Research design considerations for confirmatory chronic pain clinical trials: IMMPACT recommendations

Pain, 149 (2) (2010), pp. 177-193

[63] A.P. Association

Diagnostic and Statistical Manual of Mental Disorders

(Fifth Edition)Arlington, VA (2013)

[64] N. Bohm-Starke, U. Johannesson, M. Hilliges, E. Rylander, E. Torebjork

Decreased mechanical pain threshold in the vestibular mucosa of women using oral contraceptives: a contributing factor in vulvar vestibulitis?

J. Reprod. Med., 49 (11) (2004), pp. 888-892

[65] B.D. Reed, S.D. Harlow, L.J. Legocki, M.E. Helmuth, H.K. Haefner, B.W. Gillespie, A. Sen Oral contraceptive use and risk of vulvodynia: a population-based longitudinal study BJOG, 120 (13) (2013), pp. 1678-1684

[66] B.L. Harlow, A.F. Vitonis, E.G. Stewart

Influence of oral contraceptive use on the risk of adult-onset vulvodynia J. Reprod. Med., 53 (2) (2008), pp. 102-110

[67] M. Pollock, G. Gaesser, J. Butcher, J. Despres, R. Dishman, B. Franklin, C. Garber The recommended quantity and quality of exercise for developing and maintaining cardiorespiratory and muscular fitness, and flexibility in healthy adults

Med. Sci. Sports Exerc., 30 (1998), pp. 975-991 
[68] P. Chiarelli, J. Cockburn

Promoting urinary continence in women after delivery: randomised controlled trial

BMJ, 324 (7348) (2002), p. 1241

Corresponding author at: School of Rehabilitation, Faculty of Medicine and Health Sciences, Université de Sherbrooke, 3001, 12e avenue Nord, Sherbrooke, QC J1H 5N4, Canada.

1 The PVD Study Group includes the above authors and clinical collaborators Dr. Isabelle Girard, Dr. Yves-André Bureau, Dr. Stéphane Ouellet, Dr. Barbara Reichetzer, Dr. Laurence SimardÉmond and Dr. Ian Brochu as well as Mélanie Morin (research coordinator involved in manuscript preparation).

This is the accepted version of the following article: Morin M, Dumoulin C, Bergeron S, Mayrand MH.(2016) Randomized clinical trial of multimodal physiotherapy treatment compared to overnight lidocaine ointment in women with provoked vestibulodynia: Design and methods. Contemp Clinical Trials, 2016 Jan; 46:52-59. MS. No. Chi-15-340R1., which has been published in final form at http://ac.els-cdn.com/S1551714415301245/1-s2.0-S1551714415301245main.pdf? tid=e1510cea-6df3-11e6-b9aa$\underline{00000 a a b 0 f 6 b \& a c d n a t=1472480670 \quad 42 f 5 e b 3 a 11171 e a a 584 f 82 f c 5925 \mathrm{de} 88}$ 